\title{
Modeling Word Formation in English-German Neural Machine Translation
}

\author{
Marion Weller-Di Marco and Alexander Fraser \\ Center for Information and Language Processing \\ LMU Munich \\ \{dimarco,fraser\}@cis.uni-muenchen.de
}

\begin{abstract}
This paper studies strategies to model word formation in NMT using rich linguistic information, namely a word segmentation approach that goes beyond splitting into substrings by considering fusional morphology. Our linguistically sound segmentation is combined with a method for target-side inflection to accommodate modeling word formation. The best system variants employ source-side morphological analysis and model complex target-side words, improving over a standard system.
\end{abstract}

\section{Introduction}

A major problem in word-level approaches to MT is a lack of morphological generalization. Both inflectional variants of the same lemma and derivations of a shared word stem are treated as unrelated. For morphologically complex languages with a large vocabulary, this is problematic, especially in lowresource or domain-adaption scenarios.

A simple and widely used approach to reduce a large vocabulary in NMT is Byte Pair Encoding (BPE) (Sennrich et al., 2016), which iteratively merges the top-frequent bigrams from initially character-level split words until a set vocabulary size is reached. This strategy is effective, but linguistically uninformed and often leads to sub-optimal segmentation. Also, by only segmenting words into substrings, BPE cannot handle non-concatenative operations, for example:

- umlautung: Baum ${ }_{S g} \rightarrow$ Bäume $P l_{\text {(tree/trees), }}$

- transitional elements that frequently occur in German compounds: Grenz|kontroll|politik $\rightarrow$ Grenze, Kontrolle (border control policy)

- derivation: abundant $\leftrightarrow$ abundance

In this paper, we apply word segmentation on both the source and target sides that goes beyond merely splitting into exact substrings. This overcomes the issues caused by fusional morphology by accomodating modeling word formation across languages.

Productive word formation can lead to a high number of infrequent word forms even though the morphemes in these words are frequent. A linguistically motivated segmentation method to handle processes such as compounding and derivation allows for better coverage and generalization, both on the word level and on the morpheme level, and also enables the generation of new words. Sound morphological processing on the source and target side aims at learning productive word formation processes during translation, such as the English-German translation pair ungovernability $\leftrightarrow$ Unregierbarkeit:

$$
\text { un } \mid \text { PREF govern } \mid \text { V able } \mid \text { SUFF-ADJ ity } \mid \text { SUFF-NOUN } \leftrightarrow
$$$$
\text { un } \mid \text { PREF regieren } \mid \text { V bar } \mid \text { SUFF-ADJ keit } \mid \text { SUFF-NOUN }
$$

Morphological information should not only handle isomorphic translation equivalents as above, but also help to uncover relations between source and target side for structurally different translations.

\section{Related work}

There is a growing interest in the integration of linguistic information in NMT. For example, Eriguchi et al. (2016) and Bastings et al. (2017) demonstrate the positive impact of source-side syntactic information; Nădejde et al. (2017) report improved translation quality when using syntactic information in form of CCG tags on source and target side.

To address data sparsity, compound modeling has already been proved useful for phrase-based MT, e.g., Koehn and Knight (2003) who model source-side compounds, and Cap et al. (2014) who generate compounds on the target side. For NMT, Huck et al. (2017) apply compound and suffix segmentation using a stemmer. Ataman et al. (2017) reduce complex source-side vocabulary by means of an unsupervised morphology learning algorithm. 


\begin{tabular}{|c|c|c|c|}
\hline Morphological tag & Stem with morph. analysis & inflected form & gloss \\
\hline $\begin{array}{l}{[\mathrm{NN}-\mathrm{Neut} \cdot \mathrm{ACC} \cdot \mathrm{Pl} \cdot \mathrm{NA}]} \\
{[\mathrm{APPR}-\mathrm{ACC}]} \\
{[\mathrm{ART}-\mathrm{Fem} \cdot \mathrm{AcC} \cdot \mathrm{Sg} \cdot \mathrm{St}]} \\
{[\mathrm{NN}-\mathrm{Fem} \cdot \mathrm{AcC} \cdot \mathrm{Sg} \cdot \mathrm{NA}]} \\
{[\mathrm{ADJ}-\mathrm{NoGend} \cdot \mathrm{Gen} \cdot \mathrm{Pl} \cdot \mathrm{St}]} \\
{[\mathrm{NN}-\mathrm{Fem} \cdot \mathrm{Gen} \cdot \mathrm{Pl} \cdot \mathrm{NA}]} \\
{[\mathrm{APPR}-\mathrm{Dat}]} \\
{[\mathrm{ART}-\mathrm{Fem} \cdot \mathrm{Dat} . \mathrm{Sg} \cdot \mathrm{St}]} \\
{[\mathrm{NN}-\mathrm{Fem} \cdot \mathrm{Dat} . \mathrm{Sg} \cdot \mathrm{NA}]}\end{array}$ & $\begin{array}{l}\text { Umwelt }<\text { NN }>\text { Kriterium } \\
\text { für } \\
\text { die }<\text { Def }> \\
\text { ermitteln }<\text { V }>\text { ung }<\text { NN }><\text { SUFF }> \\
\text { Schut }<\text { NN }>\text { bedürftig }<\text { POS }> \\
\text { Meer }<\text { NN }>\text { Region } \\
\text { in } \\
\text { die }<\text { Def }> \\
\text { tief }<\text { Pos }><\text { ADJ }>\text { See }\end{array}$ & $\begin{array}{l}\text { Umweltkriterien } \\
\text { für } \\
\text { die } \\
\text { Ermittlung } \\
\text { schutzbedürftiger } \\
\text { Meeresregionen } \\
\text { in } \\
\text { der } \\
\text { Tiefsee }\end{array}$ & $\begin{array}{l}\text { ecological criteria } \\
\text { for } \\
\text { the } \\
\text { detection } \\
\text { in need of protection } \\
\text { marine regions } \\
\text { in } \\
\text { the } \\
\text { open (lit: deep) sea }\end{array}$ \\
\hline
\end{tabular}

Table 1: Representation of the training data for basic inflection prediction. Corresponding English sentence: (the ninth meeting should adopt) ecological criteria to determine marine regions in the open sea that need protection.

Ataman and Federico (2018) forego a traditional morphological analysis of the source language, and instead compose word representations from character trigrams. However, these three papers only apply segmentation on the string level and cannot properly handle fusional morphology.

Addressing morphology in NMT, Banerjee and Bhattacharyya (2018) combine BPE with a morphological analyzer to "guide" the segmentation of surface forms into substrings. Their approach does not result in morphemes, for example googling $\rightarrow$ googl|ing, which does not match with google, while in our work we match such morphemes. Tamchyna et al. (2017) present an NMT system to generate inflected forms on the target side, with a focus on overcoming data-sparsity caused by inflection. Their work contains a simple experiment on compound splitting with promising initial results that encouraged us to systematically explore word formation, including compounding, in NMT.

To model word formation, we investigate (i) source-side tags for shallow syntactic information; (ii) target-side segmentation relying on a rich morphological analysis; and (iii) source-side segmentation strategies also relying on a tool for morphological analysis. We show that combining these strategies improves translation quality.

Our contribution is a segmentation strategy that includes modeling non-concatenative processes, by implementing an English morphological analyzer suitable for this task, and by exploiting an existing tool for German, in order to obtain a consistent morphological sub-word representation.

\section{Modeling target-side morphology}

Our strategy to model word formation operates on lemma level as this allows for a better generalization than using surface forms. To model target-side inflection, we follow the simple lemma-tag generation approach by Tamchyna et al. (2017), but we improved the lemma representation to better support modeling word formation, and also implement a novel source-side morphological representation.

Lemma-tag generation (existing strategy): In a pre-processing step, all inflected forms of the target-side training data are replaced by pairs of the lemma and its rich morphological tag. In a postprocessing step, the system's output is re-inflected by generating inflected forms using the morphological tool SMOR (Schmid et al., 2004). Table 1 depicts the process of inflecting tag and lemma pairs (columns 1,2) into surface forms (column 3). New selection of lemma analyses: SMOR is a finite-state based tool covering inflection and derivation; it outputs all possible analysis paths, i.e. analyses at different levels of granularity. While not much attention is paid to the lemma selection in Tamchyna et al. (2017), a carefully selected lemmainternal representation is crucial for modeling word formation, as it provides the basis for segmentation across morphemes. To obtain optimal analyses, we follow Koehn and Knight (2003), and use the frequencies of observed non-complex words (we ignore bound morphemes). We select the analysis with the highest geometric mean of the components' frequencies, which gives a preference to words occurring more frequently in the data. The modified selection strategy favors more complex analyses; table 2 shows some examples.

\begin{tabular}{|l|l|}
\hline $\begin{array}{l}\text { Old } \\
\text { New }\end{array}$ & $\begin{array}{l}\text { atomwaffenfrei<+ADJ }> \\
\text { Atom }<\mathrm{NN}>\text { Waffe }<\mathrm{NN}>\text { frei }<+\mathrm{ADJ}> \\
\text { nuclear weapon free }\end{array}$ \\
\hline $\begin{array}{l}\text { Old } \\
\text { New }\end{array}$ & $\begin{array}{l}\text { Forschung }<\mathrm{NN}>\text { Ergebnis }<+\mathrm{NN}> \\
\text { forschen }<\mathrm{V}>\text { ung }<\mathrm{NN}><\mathrm{SUFF}>\text { Ergebnis }<+\mathrm{NN}> \\
\text { research result }\end{array}$ \\
\hline $\begin{array}{l}\text { Old } \\
\text { New }\end{array}$ & $\begin{array}{l}\text { gefährlich }<+\mathrm{ADJ}> \\
\text { Gefahr }<\mathrm{NN}>\mathrm{li} \text { Ch }<\mathrm{SUFF}><+\mathrm{ADJ}> \\
\text { danger }- \text { ous }\end{array}$ \\
\hline
\end{tabular}

Table 2: Lemma analyses with better internal analysis. Note that the derivational morpheme -ung makes the verb to research into the noun research. 


\begin{tabular}{|ll|}
\hline word & analysis \\
\hline conspiracy & conspire|V acy|SUFF/N/e \\
conspiratorial & conspire|V ator|SUFF/N/e ial|SUFF/ADJ/- \\
conspirator & conspire|V ator|SUFF/N/e \\
conspire & conspire|V \\
\hline ceremonial & ceremony|N ial|SUFF/ADJ/y \\
ceremony & ceremony|N \\
\hline acquired & acquire|V ed|SUFF/ADJ/e \\
acquirer & acquire|V er|SUFF/N/e \\
acquire & acquire|V \\
acquisition & acquire|V ition|SUFF/N/s $\rightarrow$ re \\
acquisitive & acquire|V itive|SUFF/ADJ/s $\rightarrow$ re \\
acquisitiveness & acquire|V itive|SUFF/ADJ/s $\rightarrow$ re ness $\mid \mathrm{SUFF} / \mathrm{N} /-$ \\
\hline
\end{tabular}

Table 3: English morphological analysis: the rightmost string on $S U F F$ segments denotes a string operation, such as the removal of $e$ in conspire $+a c y$.

\section{Simple English morphological analysis}

We implemented a simple morphological analyzer that is generally based on Koehn and Knight (2003), in that a word is segmented into strings that are already observed in the training data. Our method additionally relies on tag information (similar to the compound splitter of Weller-Di Marco (2017)), and on a hand-crafted set of prefixes and suffixes in combination with rules such as $i \rightarrow y$ to handle non-concatenative transitions as in beautiful $\rightarrow$ beauty $\mid N$ ful|SUFF-ADJ.

The segmentation is based on statistics derived from tagged and lemmatized data. This has several advantages: (i) the lemma and tag information restricts the possible operations (e.g., -ion as suffix is only applicable to nouns); (ii) there is no need to handle inflection; (iii) the tag provides a flat morpho-syntactic structure of the segmented word.

The analysis first identifies a potential prefix by finding a combination with a prefix in the training data, for example deactivation $\mid N \rightarrow$ de $\mid$ PREF activation $\mid N$. The tag restriction at this step is important to maintain the word class of the original word, and to avoid analyses such as decent $\mid A D J$ $\rightarrow$ de $\mid$ PREF cent $\mid N$. The remaining part undergoes splitting into either word+suffix (e.g., activation $\mid N$ $\rightarrow$ activate $\mid$ Vion $\mid S U F F-N)$ or a combination of two words (e.g., evildoer $\mid N \rightarrow$ evil $\mid N$ doer $\mid N$ ) until no further segmentation can be found. In case of several possibilities, the analysis whose components lead to the highest geometric mean is selected.

Table 3 illustrates how the morphological segmentation makes the word parts accessible such that they match with other occurrences of the word.

The splitter in its present form is rather aggressive and tends to oversplit. While it is often assumed that this is not harmful in MT (e.g., Koehn

\begin{tabular}{|l|}
\hline \multicolumn{1}{|c|}{ EN Morph-Markup - Split } \\
\hline $\begin{array}{l}\text { enthusiasm }<\mathrm{N}>\text { tic }<\text { SUFF_ADJ }>\text { ally }<\text { SUFF_ADV }> \\
\text { explode }<\text { V }>\text { ion }<\text { SUFF_N }> \\
\text { inevitable }<\text { ADJ }>\text { ly }<\text { SUFF_ADV }>\end{array}$ \\
\hline \hline \multicolumn{1}{|c|}{ EN Morph-noMarkup-Split } \\
\hline $\begin{array}{l}\text { enthusiasm tic }<\text { SUFF_ADJ }>\text { ally }<\text { SUFF_ADV }> \\
\text { explode ion }<\text { SUFF_N }> \\
\text { inevitable ly }<\text { SUFF_ADV }>\end{array}$ \\
\hline \hline \multicolumn{1}{|c|}{ EN Morph-noMarkup-noSplit } \\
\hline $\begin{array}{l}\text { enthusiasmtic }<\text { SUFF_ADJ }>\text { ally }<\text { SUFF_ADV }> \\
\text { explodeion }<\text { SUFF_N }> \\
\text { inevitablely }<\text { SUFF_ADV }>\end{array}$ \\
\hline
\end{tabular}

Table 4: Representation variants for the words enthusiastically, explosion and inevitably. This annotation replaces the lemma in the lemma-tag representation.

and Knight (2003)), we have not investigated the impact of oversplitting vs. undersplitting.

\section{Data representation in NMT}

The morphological analyses provide a straightforward basis for the segmentation experiments.

German: The lemma-tag approach (oldLemTag) is contrasted to the system variant with new lemma selection (newLemTag). For the segmentation experiments (newLemTagSplit), we apply compound splitting, such as Gold $<\mathrm{NN}>$ Preis $<\mathrm{NN}>\rightarrow$ Gold $<\mathrm{NN}>$ Preis $<\mathrm{NN}>$ (gold price). Also, nominalization, e.g., regieren $\langle\mathrm{V}>$ ung $\langle\mathrm{NN}\rangle\langle\mathrm{SUFF}>$ (govern ment), is segmented, but different adjective suffixes (such as -lich) are kept attached. Generally, we found that variation of the splitting granularity of adjective suffixes does not have a large impact.

English: We first look at a representation where lemma-tag pairs replace surface forms (LemTag). To evaluate the effect of morphological information, we compare the three settings in table 4 that also rely on the lemma-tag representation: the tags convey inflectional information, but the lemma is replaced by its morphological analysis.

In Morph-Markup-Split, words are split following the analysis, with tags indicating word-internal structure. Morph-noMarkup-Split is the same, but without word-internal tags. The annotation of prefixes/suffixes (-ion $<S U F F-N>$ ) is always kept.

In addition to explicit splitting, we consider a variant where lemmas are replaced by the unsplit morphological analysis (Morph-noMarkupnoSplit), and all segmentation is done by BPE, which can now access actual words (enthusiasm instead of *enthusias) that already occur in the training data. This representation is conceptionally similar to the German lemma-tag representation. 


\begin{tabular}{|c|l|l|l|c|c|c|}
\hline System & Source (EN) & Target (DE) & Small & Medium & Large2M & Large4M \\
\hline 1 & plain & plain & 21.77 & 26.60 & 28.66 & 33.71 \\
\hline 2 & plain & oldLemTag & 22.25 & 26.96 & 28.87 & 33.97 \\
3 & plain & newLemTag & 22.47 & 27.05 & 28.61 & 33.90 \\
\hline 4 & LemTag & newLemTag & 23.32 & 27.36 & 28.88 & 34.28 \\
5 & LemTag & newLemTagSplit & 22.55 & 27.22 & 29.07 & 34.21 \\
\hline 6 & LemTag MorphMarkup-Split & newLemTag & 21.85 & 26.90 & 29.33 & 33.96 \\
7 & LemTag Morph-noMarkup-Split & newLemTag & 22.86 & 27.05 & 29.20 & 34.10 \\
8 & LemTag Morph-noMarkup-noSplit & newLemTag & 22.82 & 27.18 & 29.18 & 34.12 \\
\hline 9 & LemTag MorphMarkup-Split & newLemTagSplit & 22.25 & 27.12 & 29.39 & 34.38 \\
10 & LemTag Morph-noMarkup-Split & newLemTagSplit & 22.53 & 26.90 & 29.10 & 34.12 \\
11 & LemTag Morph-noMarkup-noSplit & newLemTagSplit & 23.13 & 27.55 & 29.42 & 34.19 \\
\hline
\end{tabular}

Table 5: Experimental results in case-sensitive BLEU for 4 training settings.

\section{Experiments}

We compare four training settings: small $(248,730$ sentences: news-commentary), large $2 M(1,956,444$ sentences: Europarl + news-commentary), large $4 M$ $(4,116,215$ sentences: Europarl + news-commentary + CommonCrawl) and medium (1M sentences) where the medium corpus consists of the newscommentary corpus and the first $\sim 750 \mathrm{k}$ sentences of Europarl. We use WMT'15 as dev-set (2169 sentences) and WMT' 16 as test-set (2999 sentences).

The lemma-tag approach doubles the sentence length by inserting tags. To avoid overly long sentences, the training data was first filtered to sentences of length 50, and after that, sentences more than 60 words long after BPE splitting were removed (e.g., sentences containing mostly foreign language words split nearly at character level).

Data pre-processing The baseline was trained on plain surface forms (tokenized and true-cased).

For the German lemma-tag system, we used BitPar (Schmid, 2004) to obtain morphological features in the sentence context, and SMOR (Schmid et al., 2004) for morphological analysis. For English, we used TreeTagger (Schmid, 1994). The English morphological analyzer for the small, medium and large $2 \mathrm{M}$ systems was trained on the large2M data, the analyzer for the large $4 \mathrm{M}$ system was trained on the full $\sim 4 \mathrm{M}$ lines.

All systems (baseline and lemma-tag variants) underwent BPE segmentation ("joint" BPE of source/target side) with 30k merging operations.

\begin{tabular}{|ll|ll|}
\hline encoder & transformer & initial-learning-rate & 0.0002 \\
decoder & transformer & label-smoothing & 0.1 \\
batch-type & word & transf.-dropout-act & 0.1 \\
batch-size & 4096 & transf.-dropout-attention & 0.1 \\
num-layers & 6 & transf.-dropout-prepost & 0.1 \\
max-seq-len & $\max$ sent len & checkpoint-frequency & 3000 \\
\hline
\end{tabular}

Table 6: Sockeye parameters for the Transformer model.
Training For the experiments, we used a Transformer NMT model (Sockeye toolkit: Hieber et al. (2017)). Table 6 shows the training parameters.

\subsection{System variants}

Table 5 shows different representation variants on the source and target side, as outlined in section 5 . Generally, the lemma-tag systems are better than a standard NMT system; there is not much difference between the old (Tamchyna et al., 2017) and the new version (lines 2 and 3). Source-side lemma-tag pairs improve the small and medium settings when paired with non-split German data; split German data works better for the Large2M system. Both variants perform similarly for the Large $4 \mathrm{M}$ system (lines 4 and 5). English word-internal markup improves the Large2M system, both with split and unsplit German data (lines 6 and 9), and leads to the best result when combined with split German data in the Large4M setting (line 9). The variants in lines 7 and 8 (split/unsplit morphological analysis) produce similar results when translating to non-split German data. Interestingly, with explicit splitting on the German side (lines 10 and 11), the non-split English data performs considerably better for the small/medium/large2M settings, leading to the best results overall for these data settings.

There seems to be a tendency that explicit splitting on both sides harms the smaller settings, possibly because translating at morpheme level requires more training data. Similarly, the English wordinternal markup might introduce a complexity that only the larger systems can handle. On the other hand, using the non-split morphological analysis is less intrusive, but potentially useful at the BPE segmentation step by providing better access to sub-words. However, the best variants use explicit segmentation on the target side - this makes the question "to split or not to split" difficult to answer. 


\begin{tabular}{|c|c|c|c|c|}
\hline System & Small & Medium & Large2M & Large4M \\
\hline 1 & 17.17 & 22.12 & 24.32 & 30.85 \\
\hline 2 & 18.86 & 22.85 & 25.13 & 31.67 \\
3 & 19.16 & 23.13 & 24.46 & 31.52 \\
\hline 4 & 19.30 & 22.75 & 24.42 & 31.70 \\
5 & 18.45 & 23.05 & 24.68 & 32.56 \\
\hline 6 & 18.19 & 22.92 & 24.93 & 32.23 \\
7 & 19.43 & 23.06 & 25.22 & 31.74 \\
8 & 18.79 & 22.55 & 24.68 & 32.15 \\
\hline 9 & 18.79 & 22.67 & 24.68 & 31.95 \\
10 & 18.06 & 22.44 & 24.98 & 32.25 \\
11 & 18.75 & 23.09 & 24.46 & 31.99 \\
\hline \multicolumn{4}{|l}{} \\
\hline
\end{tabular}

Table 7: BLEU scores for a medical-domain test set.

Maybe always splitting at a certain level is not the right approach, but rather a more context-sensitive segmentation strategy would be desirable.

\subsection{Application to out-of-domain data}

In low-resource scenarios, such as translating data of a particular domain, the problems caused by inflectional variants and forms created through derivation are typically aggravated. Applying a system trained on general language, but with a component to handle inflection and word formation, to an outof-domain test set constitutes an interesting use case. We use a test set ${ }^{1}$ (Haddow et al., 2017) from the medical domain (1931 sentences), containing health information aimed at the general public and summaries of scientific studies. Table 7 shows the results for the different system variants. For all data settings, the lemma-tag variants are better than the surface form baselines. There are no clear tendencies for a best-performing strategy across all settings, but English morphological analysis seems to contribute less, whereas English lemma-tag information (lines 4,5 ) leads to overall good results.

\subsection{Examples}

Table 8 shows two examples to illustrate the effect of morphological analysis. In (a), the baseline translates the noun foolishness as adjective, whereas the morphologically enriched system chooses a valid translation. Looking at the representation of foolishness after BPE segmentation, the baseline's fool@@is@@hn@@ess is not particularly meaningful, whereas the representation of system 6 is [NN] fool $<\mathrm{N}>i$ sh $<$ SUFF_ADJ $>$ ness $<$ SUFF_N $>$, which provides a better basis for translation.

In (b), from the medical test set, the baseline fails to translate coagulation. Below, the BPE representations of coagulation $(\mathrm{f}=19)$ and coagulate

\footnotetext{
${ }^{1}$ HimL-testset-2015 from www. himl . eu/test-sets
}

\begin{tabular}{|l|l|}
\hline In & $\begin{array}{l}\text { For all his foolishness Ed Miliband knew who his } \\
\text { enemies were. }\end{array}$ \\
\hline S1 & $\begin{array}{l}\text { Trotz seines törichten Ed Miliband wusste er, wer seine } \\
\text { Feinde waren. } \\
\text { For his foolish Ed Miliband knew he, who his enemies were. }\end{array}$ \\
\hline S6 & $\begin{array}{l}\text { Trotz all seiner Dummheit wusste Ed Miliband, wer seine } \\
\text { Feinde waren. }\end{array}$ \\
\hline Ref & $\begin{array}{l}\text { Bei all seiner Verrücktheit wusste Ed Miliband, wer seine } \\
\text { Feinde waren. }\end{array}$ \\
\hline In & $\begin{array}{l}\text { Current testing for TIC normally involves coagulation tests } \\
\text { on the patient's blood. }\end{array}$ \\
\hline S1 & $\begin{array}{l}\text { Aktuelle Tests für TIC beinhalten normalerweise } \\
\text { Coagulationstests am Blut des Patienten. }\end{array}$ \\
\hline S10 & $\begin{array}{l}\text { Bei der aktuellen TIC-Prüfung handelt es sich in der Regel } \\
\text { um Gerinnungstests am Blut des Patienten. }\end{array}$ \\
\hline Ref & $\begin{array}{l}\text { Aktuelle Erprobung von TIC beinhaltet normalerweise } \\
\text { Gerinnungstests der Blut des Patienten. }\end{array}$ \\
\hline
\end{tabular}

Table 8: Translation examples from the Large2M setting (a) and the Large $4 \mathrm{M}$ setting (b).

$(\mathrm{f}=3)$ in the respective systems are compared:

\begin{tabular}{|l|ll|}
\hline Surface (System 1) & Tag & morph. annotated (System 10) \\
\hline co@@ag@@ ulation & {$[\mathrm{NN}]$} & co@@ ag@ @ ulate ion $<$ SUFF_N $>$ \\
co@@ag@@ alate & [VB] & co@@ag@@ ulate \\
\hline
\end{tabular}

Even with BPE segmentation, the representation in System 10 is more general than in the surface system, and in particular allows matching with e.g., coagulate. Similarly, Gerinnungstest (coagulation test) is represented as ger@ @innen $<\mathrm{V}>$ ung $<\mathrm{NN}><\mathrm{SUFF}>$ Test $<\mathrm{NN}>$, allowing to combine statistics of the verb gerinnen and the noun Gerinnung. Thus, better generalization, paired with tag information, enables the morphology-informed systems to make better use of the training data.

\section{Conclusion}

We showed that morphologically sound segmentation that considers non-concatenative processes in order to obtain a consistent representation of subwords improves translation. The findings of our experiments provide important insights for translating morphologically rich languages, and are particularly important for low-resource settings.

\section{Acknowledgements}

This research was partially funded by LMU Munich's Institutional Strategy LMUexcellent within the framework of the German Excellence Initiative. This project has received funding from the European Research Council (ERC) under the European Union's Horizon 2020 research and innovation programme (grant agreement № 640550). This work was supported by the Dutch Organization for Scientific Research (NWO) VICI Grant nr. 277-89-002. 


\section{References}

Duygu Ataman and Marcello Federico. 2018. Compositional Representation of Morphologically-Rich Input for Neural Machine Translation. In Proceedings of the 56th Annual Meeting of the Association for Computational Linguistics (Short Paper), pages 305-311, Melbourne, Australia.

Duygu Ataman, Matteo Negri, Marco Turchi, and Marcello Federico. 2017. Linguistically Motivated Vocabulary Reduction for Neural Machine Translation from Turkish to English. In Proceedings of EAMT 2017, pages 331-342, Prague, Czech Republic.

Tamali Banerjee and Pushpak Bhattacharyya. 2018 Meaningless yet meaningful: Morphology grounded subword-level NMT. In Proceedings of the Second Workshop on Subword/Character LEvel Models, pages 55-60, New Orleans. Association for Computational Linguistics.

Joost Bastings, Ivan Titov, Wilker Aziz, Diego Marcheggiani, and Khalil Sima'an. 2017. Graph Convolutional Encoders for Syntax-Aware Neural Machine Translation. In Proceedings of EMNLP 2017, pages 1957-1967, Copenhagen, Denmark.

Fabienne Cap, Alexander Fraser, Marion Weller, and Aoife Cahill. 2014. How to Produce Unseen Teddy Bears: Improved Morphological Processing of Compounds in SMT. In Proceedings of the 14th Conference of the European Chapter of the Association for Computational Linguistics, pages 579-587, Gothenburg, Sweden. Association for Computational Linguistics.

Akiko Eriguchi, Kazuma Hashimoto, and Yoshimasa Tsuruoka. 2016. Tree-to-Sequence Attentional Neural Machine Translation. In Proceedings of $A C L$ 2016, pages 823-833, Berlin, Germany.

Barry Haddow, Alexandra Birch, Ondřej Bojar, Fabienne Braune, Colin Davenport, Alex Fraser, Matthias Huck, Michal Kašpar, Květoslava Kovař́ková, Josef Plch, Anita Ramm, Juliane Ried, James Sheary, Aleš Tamchyna, Dušan Variš, Marion Weller, and Phil Williams. 2017. HimL: Health in my Language. In Proceedings of the EAMT 2017 User Studies and Project/Product Descriptions, page 33, Prague, Czech Republic.

Felix Hieber, Tobias Domhan, Michael Denkowski, David Vilar, Artem Sokolov, Ann Clifton, and Matt Post. 2017. Sockeye: A Toolkit for Neural Machine Translation. arXiv preprint arXiv:1712.05690.

Matthias Huck, Simon Riess, and Alexander Fraser. 2017. Target-side Word Segmentation Strategies for Neural Machine Translation. In Proceedings of the Second ACL Conference on Machine Translation (WMT), pages 56-67, Copenhagen, Denmark.

Philipp Koehn and Kevin Knight. 2003. Empirical Methods for Compound Splitting. In Proceedings of the 10th Conference of the European Chapter of the Association for Computational Linguistics, pages 187-194, Budapest, Hungary. Association for Computational Linguistics.

Maria Nădejde, Siva Reddy, Rico Sennrich, Tomasz Dwojak, Marcin Junczys-Dowmunt, Philipp Koehn, and Alexandra Birch. 2017. Syntax-Aware Neural Machine Translation Using CCG. CoRR, abs/1702.01147.

Helmut Schmid. 1994. Probabilistic Part-of-Speech Tagging Using Decision Trees. In International Conference on New Methods in Language Processing, pages 44-49, Manchester, UK.

Helmut Schmid. 2004. Efficient Parsing of Highly Ambiguous Context-Free Grammars with Bit Vectors. In Proceedings of the International Conference on Computational Linguistics, pages 162-168, Geneva, Switzerland.

Helmut Schmid, Arne Fitschen, and Ulrich Heid. 2004. SMOR: A German Computational Morphology Covering Derivation, Composition, and Inflection. In Proceedings of the 4th International Conference on Language Resources and Evaluation (LREC), pages 1263-1266, Lisbon, Portugal.

Rico Sennrich, Barry Haddow, and Alexandra Birch. 2016. Neural Machine Translation of Rare Words with Subword Units. In Proceedings of the 54th Annual Meeting of the Association for Computational Linguistics, ACL 2016.

Aleš Tamchyna, Marion Weller-Di Marco, and Alexander Fraser. 2017. Modeling Target-Side Inflection in Neural Machine Translation. In Proceedings of the Second ACL Conference on Machine Translation (WMT), pages 32-42, Copenhagen, Denmark.

Marion Weller-Di Marco. 2017. Simple Compound Splitting For German. In Proceedings of the 13th Workshop on Multiword Expressions (MWE17), Valencia, Spain. 